\title{
Exclusive Breastfeeding: Dream to Achieve, Hurdles to Overcome
}

\author{
Nisha Nair ${ }^{1}$ \\ ${ }^{1}$ California State University, Fresno, USA \\ Correspondence: Nisha Nair, California State University, Fresno, USA.
}

Received: September 20, 2019

doi:10.20849/ijsn.v4i4.658

\author{
Accepted: October 1, $2019 \quad$ Online Published: October 11, 2019 \\ URL: https://doi.org/10.20849/ijsn.v4i4.658
}

\begin{abstract}
The pattern of infant feeding during the first six months has a significant influence on the infant's health outcomes. Unfortunately, many mothers find it difficult to satisfy their goal of exclusive breastfeeding, despite a higher rate in the initiation of breastfeeding. This narrative review aims to explore the literature for barriers linked with exclusive breastfeeding. Using appropriate search terms, the scholarly database was searched for relevant articles that met the review objective. The preponderance of current evidence strongly suggests that exclusivity in breastfeeding is possible by taking steps to reduce the barriers faced by the mothers. This article will provide an overview for nurses who care for women during the intrapartum and postpartum period to understand the obstacles and to promote breastfeeding self-efficacy and exclusiveness.
\end{abstract}

Keywords: barriers, exclusive breastfeeding, duration, weaning, breastfeeding support

\section{Introduction}

Breastfeeding is viewed as the gold standard in newborn feeding, as it provides optimum nutrition for babies. The World Health Organization (2016) and the American Academy of Pediatrics (2012) unanimously set guidelines to recommend exclusive breastfeeding from birth until 6 months. In addition to the nutritional advantages, breastfeeding provides an array of benefits for the mother and infant.

The AAP recommends exclusive breastfeeding for about the first 6 months of life, with continued breastfeeding as appropriate complementary foods are introduced. The WHO similarly recommends that infants be exclusively breastfed for the first 6 months, with breastfeeding continuing for up to 2 years or beyond, as complementary foods are added. Exclusive breastfeeding for the first six months is also recommended by other professional health care organizations, such as the American Academy of Family Physicians (AAFP, 2012), the American College of Nurse-Midwives (ACNM, 2016), the Academy of Breastfeeding Medicine (Chantry, Eglash, \& Labbok, 2015), and the American College of Obstetricians and Gynecologists (ACOG, 2018). The Association of Women's Health, Obstetric, and Neonatal Nurses (AWHONN, 2014; 2015) strongly supports breastfeeding as the ideal nutrition for infants and provides policies and guidelines for nurses in promoting and supporting breastfeeding families.

\section{Background/Current Situation}

Breastfeeding rates in the United States have been increasing steadily over the past decade. The Centers for Disease Control and Prevention (CDC, 2018) reported that the U.S. breastfeeding initiation rate for infants born in 2013 was $81.1 \%$, which is the highest ever reported. The 6-month breastfeeding rate was $51.8 \%$ and the 12 -month rate was $30.7 \%$. The rate of exclusive breastfeeding at 3 months was $44.4 \%$ and, at 6 months, $22.3 \%$. With the increase in breastfeeding rates, the U.S. is close to meeting the Healthy People 2020 goal of $81.9 \%$ of infants ever breastfed. More than $50 \%$ of states have already met or exceeded this goal. The U.S. continues to fall short of meeting Healthy People goals of $60.6 \%$ breastfeeding at 6 months, and $34.1 \%$ at 12 months, as well as goals for exclusive breastfeeding of $46.2 \%$ through 3 months and 25.5\% through 6 months (CDC, 2018; U.S. Department of Health and Human Services [USDHHS], 2016). The CDC (2016) suggests that the low breastfeeding continuation rates may be related to lack of support offered to breastfeeding mothers by family members, health care providers, and employers. Although the recommendations are well known, mothers are facing several barriers that make the exclusive breastfeeding difficult. 


\section{Barriers}

Recent studies show a great increase in mothers understanding the benefits of breastfeeding, but the actual challenge lies in the continuation of breastfeeding for at least six months. The decisions to breastfeed and persist in breastfeeding are influenced by various factors, including breastfeeding issues, maternal, confidence, adequate social support, cultural factors, breastfeeding education, exposure to other mothers breastfeeding, and hospital practices (Johnson, Kirk, \& Muzik, 2015). It is vital that nurses understand these barriers to better support the mothers.

\subsection{Sociodemographic Reasons}

\subsubsection{Maternal Employment/Return to Work}

Returning to work within the first 12 weeks of delivery can adversely affect the intention of breastfeeding duration. Some mothers doubt their abilities to continue breastfeeding if they no longer have immediate access to their infants. On the other hand, some mothers are uncertain about continuing breastfeeding since they fear not finding enough time or a convenient location to express milk during their workdays. Reported barriers to breastfeeding successfully in the workplace include nonsupportive workplace, lack of compliance with breastfeeding laws, lack of support from coworkers and supervisors, inadequate breastfeeding information, and a lack of paid maternity leave (Johnson et al., 2015; Kim, Fiese, \& Donovan, 2017; Majee, Jefferson, Goodman, \& Olsberg, 2016).

Each country has its own regulations for maternity and paternity leave. Mothers in the U.S. often return to work earlier so that they can continue to provide financially for their families. This leaves mothers with less time to breastfeed.

\subsubsection{Geographical (Urban VS Rural)}

Rural areas are affected by geographic isolation, decreased population, low income, and difficulty in accessing health services. In the U.S., breastfeeding initiation and continuation rates are lower in rural areas than in urban areas, with rates of initiation $18 \%$ lower in rural areas (Sparks, 2010). The situation is similar in other industrialized countries, like the United Kingdom and Australia. In the United Kingdom, breastfeeding initiation in rural areas are lower than the average national rates. The Australian National Infant Feeding Survey also found that the rate of breastfeeding initiation rates was lower in remote locations than in their major cities (Stamp \& Casanova, 2006).

\subsubsection{Race/Ethnicity}

A growing body of research has highlighted the relationship between race and ethnicity with breastfeeding rates. Non-White women who migrate to the U.S. are more likely to breastfeed than are women of the same ethnicity born in the U.S. (Hawkins, Gillman, Shafer, \& Cohen, 2014; Sparks, 2011). Similarly, Hispanic women in the U.S. also exhibit this trend. Acculturation has a role in people's health practices. Interestingly, studies show that Hispanics who primarily speak Spanish are less acculturated and those who primarily speak English are considered more acculturated. Comparing to more acculturated Hispanic women, less acculturated Hispanic women are more likely to breastfeed at all and exclusively, and tend to breastfeed longer (Ahluwalia, D'Angelo, Morrow, \& McDonald, 2012; Chapman \& Perez Escamilla 2013; Wambach, Domian, Page-Goertz, Wurtz, \& Hoffman, 2016). In another study, Black women had less proximity to hospitals implementing breastfeeding support services and were more likely to be given infant formula in the hospital (Lind, Perrine, Li, Scanlon, \& Grummer-Strawn, 2014; McKinney et al., 2016).

\subsubsection{Marital Status}

There is a higher rate of exclusive breastfeeding in the hospital among married women, as well as among women with higher socioeconomic status. It is possible that, compared to unmarried women, married women may benefit from greater financial security and encouragement and support for breastfeeding, especially that which is provided by their partners (Perrine, Scanlon, Li, Odom, \& Grummer-Strawn, 2012). At the same time, unmarried women may be conducting all household activities alone, along with infant care. Unmarried women may feel overwhelmed and inclined to use ready-made formula bottles over the struggle of establishing breastfeeding (Rempel \& Rempel, 2011).

\subsubsection{Age}

Maternal age is also one of the numerous factors that shows a relationship with success in breastfeeding rates. Reports show that women who are under 20 years of age have decreased initiation or shorter durations of breastfeeding as compared to their older counterparts (Apostolakis-Kyrus, Valentine, \& Defranco, 2013; Sipsma, Magriples, Gordon, Gabzdyl, \& Kershaw, 2013). In 2010, 6\% of infants born to women younger than 20 years 
were exclusively breastfed at six months, which is almost half the rate for infants born to women 30 years or older (USDHHS), 2016). Barriers may include the physical discomfort associated with lactation or an overall lack of intention to breastfeed resulting from plans to return to school or work, the stigma associated with breastfeeding in public, or the influence of a dissuasive social support system (Taveras et al., 2013). Adolescent mothers are also less likely to breastfeed and those who start breastfeeding are likely to stop within 1 month postpartum (Kanhadilok \& McGrath, 2015).

\subsection{Health Care Related Reason}

\subsubsection{Mode of Birth}

Mode of delivery is highlighted as a major factor in dropping out of exclusive breastfeeding. Hedburg (2013) found a correlation between Cesarean delivery and unfavorable practices related to breastfeeding. Delayed skin to skin contact between mother and baby, missing the golden hour in breastfeeding initiation, higher incidence of supplemental feeding, and separation of mother and baby all can lead to nonexclusive breastfeeding. Ongoing efforts to promote normal vaginal deliveries may have a positive impact on breastfeeding outcomes.

\subsubsection{Formula Supplementation}

A strong association between exclusive breastfeeding during the postpartum phase in hospital and exclusive breastfeeding duration has been well established in various studies (DiGirolamo, Grummer-Strawn, \& Fein, 2008). Several hospitals have well-written policies that require the use of formula supplementation of breastfed infants only for a valid medical indication or because of maternal request after teaching (Kanhadilok \& McGrath, 2015; Mueffelmann, Racine, Warren-Findlow, \& Coffman, 2015; Odom, Li, Scanlon, Perrine, \& Grummer-Strawn, 2014; Roll \& Cheater, 2016). Consequently, in-hospital use of formula supplements for breastfed newborns is largely by maternal request, particularly among low-income mothers who have not attended prenatal breastfeeding classes (Roll \& Cheater, 2016). A survey of low income, African American mothers found that $60 \%$ initiated breastfeeding and $78 \%$ of these breastfed newborns received formula supplementation in the hospital, predominantly because of maternal request (Lind et al., 2014; McKinney et al., 2016). Although bedside nurses recognize that formula use should be limited, knowledge gaps in current guidelines and lack of teaching time; maternal complaints of fatigue; insufficient milk supply or sore nipples; and infant challenges, such as fussiness, sleepiness, or latch difficulties contribute to formula use on the part of nursing staff. Because mothers tend to continue infant care practices begun in the hospital, those used to hospital formula supplements require close follow-up and monitoring to transition to full breastfeeding as quickly as possible (Rosen-Carole, Hartman, \& Academy of Breastfeeding Medicine, 2015).

\subsubsection{Prenatal Education}

Prenatal education increases the rate of breastfeeding initiation, duration, and exclusivity. Effective interventions include easy access to informational materials and interpersonal support. Support and teaching from an International Board Certified Lactation Consultant (IBCLC), peer counselor, health care provider, and bedside nurse is essential for the success of breastfeeding moms. Numerous systematic reviews showed that health education and peer support interventions increased breastfeeding initiation and duration (Dyson, McCormick, \& Renfrew, 2005; Lumbiganon et al., 2012). Interpersonal support like home visits, telephone hotlines, and resource centers allowed mothers to communicate in real-time to solve breastfeeding issues. Lack of access to breastfeeding education during home visits and prenatal clinics is also a significant barrier to successful breastfeeding (Lumbiganon et al., 2012).

\subsubsection{High Maternal Body Mass Index (BMI)}

Studies show a direct relationship between maternal weight and infant feeding decisions. Maternal BMI decreases breastfeeding rates. Women with BMIs of 30 or more are less likely to initiate breastfeeding and have shorter durations of breastfeeding (Lepe, Bacardí Gascón, Castañeda-González, \& Pérez Morales, 2011; Turcksin, Bel, Galjaard, \& Devlieger, 2014). They also have psychosocial characteristics associated with poor breastfeeding outcomes and are at risk for delayed onset of lactation (Turcksin et al., 2014). Providing breastfeeding care may be more challenging due to anatomical and physical mobility issues, as well as modesty and the stigma associated with being obese. Women with higher BMIs can be referred for expert breastfeeding education prenatally and provided breastfeeding support and management postnatally to help them meet their goals. For mothers who have undergone bariatric surgery and plan to breastfeed, nutritional deficiencies can be a barrier. These mothers may benefit from seeing a registered dietician and lactation consultant during pregnancy and postpartum to optimize effective breastfeeding (Caplinger et al., 2015). 


\subsubsection{Maternal Substance Use}

For mothers with substance use disorders, especially those with opioid addictions, the challenges of breastfeeding can be innumerable. The infants' experiences of neonatal abstinence syndrome (NAS) or other neuro behaviors related to in utero exposures can affect lactation (Jansson \& Velez, 2015). For example, hypertonicity and uncontrolled movements caused by NAS can create difficulty in positioning the infant on the breast. Poor sleep/awake state control, sensory (including oral) hypersensitivity, and feeding disturbances, such as suck/swallow incoordination are common in these infants, which can make breastfeeding a greater challenge (Reece-Stremtan, Marinelli, \& ABM, 2015).

\subsubsection{Prior Breastfeeding Experience}

Women tend to select the same method of infant feeding for each of their children. If the first child was breastfed, there is a higher chance that subsequent infants will be breastfed. Brief periods of past breastfeeding and negative experiences can adversely affect future breastfeeding. In the same vein, positive experiences can improve attitudes, confidence, self-efficacy, motivation, and intention to breastfeed (Huang, Ouyang, \& Redding, 2019).

\subsubsection{Provider Role}

Health care providers and nurses play key roles in infant feeding decisions, as well as in the promotion of exclusive breastfeeding. Strategies that promote breastfeeding in the prenatal setting include a breastfeeding-friendly office or clinic environment; intentional promotion, education, and support for breastfeeding throughout prenatal care; and discussion of breastfeeding at each prenatal visit (Rosen-Carole et al., 2015). Taking advantage of training opportunities to improve breastfeeding knowledge, problem-solving, and counseling can increase nurses' effectiveness and comfort in providing breastfeeding management and support (Labarere et al., 2005). A targeted breastfeeding course is integral in the nursing curriculum to increase nurses' knowledge, confidence, and techniques to promote exclusive breastfeeding. A study by Feldman-Winter, Barone, and Milcarek (2010) found that gaps in breastfeeding knowledge, training, and counseling skills among health care providers involved with breastfeeding mothers had deleterious effects on decisions for exclusive breastfeeding.

\subsubsection{Hospital Practices}

Hospitals that promote the continuous rooming-in of mother and baby created conducive environments that encouraged new mothers to continue skin-to-skin contact and uninterrupted breastfeeding (DiGirolamo et al., 2008). Rooming-in also helped mothers to recognize and respond to their infants' feeding cues, become comfortable latching their babies and breastfeeding, and gain confidence in their abilities to care for their infants. However, these potential benefits of rooming-in can be interrupted with frequent visitations from guests. The constant presence of visitors can interfere with skin-to-skin contact, unwittingly encourage parents to start using pacifiers while guests are present, and hinder the lactation consultant from offering one-on-one instruction. Nurses who offer to care for babies in the nurseries so their mothers can rest also increase the risk of formula supplementation. Many hospitals have implemented a designated afternoon "quiet time" or "nap time" to give mothers more time alone with their newborns.

\subsection{Psychosocial Reasons}

\subsubsection{Low Maternal Confidence}

Breastfeeding initiation, duration, and exclusiveness also depend on the psychology factor related to maternal confidence. Several researchers highlighted the fact that mothers who were more confident about the whole process of breastfeeding were more likely to have longer breastfeeding duration and higher rates of exclusive breastfeeding (de Jager et al., 2015; Khan, Vesel, Bahl, \& Martines, 2015). Therefore, nurses who work with mothers and families need to promote maternal confidence and self-efficacy to progress toward the goal of exclusive breastfeeding for the first six months.

\subsubsection{Maternal Depression and Anxiety}

Maternal anxiety can be one of the greatest predictors of breastfeeding discontinuation in the first 6 months postpartum (Adedinsewo, Fleming, Steiner, Meaney, \& Girard, 2012). Mothers who are having anxiety issues often struggle to overcome the difficulty in measuring adequate milk intake with each feeding (Arifunhera et al., 2016). The role of the bedside nurse is crucial to building confidence about the adequacy of breastfeeding. Sankar et al. (2015) found that mothers were less likely to initiate breastfeeding and more likely to discontinue breastfeeding early if they were depressed at any point during the pregnancy or in the first 6 weeks postpartum. When an increased risk of depression is noted during a prenatal visit, or in a woman who has just given birth, it is 
essential for the health care provider to discuss the benefits of breastfeeding for the health of the infant and the mother.

\subsubsection{Maternal Attitudes}

The mother's perception and attitudes regarding breastfeeding can impact her choice for exclusive breastfeeding. Some mothers view breastfeeding as more convenient, while others choose to use formula so other family members can help with feedings. Breastfeeding can be viewed as time-consuming by some mothers since they see formula-feeding as having fewer restrictions on family and social life (Hedburg, 2013; Roll \& Cheater, 2016). Certain mothers initiate breastfeeding in response to pressure from family members, health care professionals, or their own perceptions of being "good mothers." These mothers may say they plan to "try breastfeeding," while lacking commitment and determination (Roll \& Cheater, 2016). These women benefit from care by bedside nurses who will help them explore their feelings and concerns and provide information, support, and assistance.

\subsubsection{Support Person}

Partner and family support are major factors in the mother's decision to breastfeed. Health professionals should educate fathers about ways to support their partners with the goal of exclusive breastfeeding. Partners are considered crucial members of the mother's "breastfeeding supporting team," providing physical help and emotional support (Roll \& Cheater, 2016). Grandmothers also have a great impact on a mom's decision to breastfeed.

\section{Implications for Practice}

By consistently addressing these barriers, breastfeeding success rates can be significantly increased. Evidence-based information and support need to be given by all health care providers to families to achieve their breastfeeding goals. The interdisciplinary team itself can be included in the care which comprises obstetricians, midwives, and nurse practitioners providing prenatal education; labor and delivery nurses and postpartum nurses should continue providing support; and lactation consultants, pediatricians, and everyone else involved in the team should be giving the same information. The infants will be healthier, the families will be happier, and in turn, will help to meet the Healthy People 2020 goal of $81.9 \%$ of infants ever breastfeed.

First, society should support mothers who are breastfeeding by ensuring a supportive workplace and complying with breastfeeding laws. Advocacy efforts should also aim at providing paid maternity leave for breastfeeding mothers to continue bonding without the added stress of returning to work early due to financial obligations. In terms of prenatal education, public awareness and educational campaigns should focus on the positive health outcomes associated with breastfeeding with easy access to informational materials and interpersonal support. Health care providers could also benefit from training opportunities to improve breastfeeding knowledge. Lastly, access to support groups should be offered in a variety of formats (in-person and on-line) and should act as a platform to address the various barriers to sustain breastfeeding exclusivity.

\section{Conclusion}

Understanding exclusive breastfeeding barriers can assist in identifying gaps in the support process, with the ultimate goal of increasing breastfeeding success and reducing the incidence of early weaning. Most mothers and infants are not receiving the health benefits associated with exclusive breastfeeding (Odom et al., 2013). In all the studies, the role of health care practices in initiating breastfeeding, duration, and exclusivity are emphasized. All bedside nurses need to increase their own breastfeeding knowledge, problem-solving skills, and counseling, as well as work closely with their lactation consultants to promote exclusive breastfeeding for the first six months. Healthcare providers, with an awareness of these barriers, can work in communities, at their facilities, in their practices, and individually with mothers to help them feel more prepared and supported so that they can more easily reach their breastfeeding goals. When these challenges are effectively identified and addressed, breastfeeding rates will likely rise, thereby improving the health of women and infants.

\section{References}

Adedinsewo, D., Fleming, A., Steiner, M., Meaney, M., \& Girard, A. (2012). The effect of maternal anxiety/depression on breastfeeding outcomes: MAVAN (Maternal Adversity Vulnerability and Neurodevelopment) Study. Retrieved from https://www.fasebj.org/doi/abs/10.1096/fasebj.26.1_supplement.368.3

Ahluwalia, I. B., D'Angelo, D., Morrow, B., \& McDonald, J. A. (2012). Association between acculturation and breastfeeding among Hispanic women: Data from the Pregnancy Risk Assessment and Monitoring System. Journal of Human Lactation, 28(2), 167-173. 
American Academy of Family Physicians. (2012). Breastfeeding Policy Statement. Retrieved from http://www.aafp.org/online/en/home/policy/policies/b/breastfeedingpolicy.html

American Academy of Pediatrics Section on Breastfeeding. (2012). Breastfeeding and the use of human milkPolicy statement. Pediatrics, 129(3), e827-e841.

American College of Nurse-Midwives. (2016). Position Statement: Breastfeeding. Retrieved from http://www.midwife.org/ACNM/files/ACNMLibraryData/UPLOADFILENAME/000000000248/Breastfeed ing-statement-Feb-2016.pdf

American College of Obstetricians and Gynecologists. (2018). Committee opinion no. 756: Optimizing support for breastfeeding as part of obstetric practice. Obstetrics and Gynecology, 132(4), e187-e196.

Apostolakis-Kyrus, K., Valentine, C., \& Defranco, E. (2013). Factors associated with breastfeeding initiation in adolescent mothers. Journal of Pediatrics, 163(5), 1489-1494.

Arifunhera, J. H., Srinivasaraghavan, R., Sarkar. S., Kattimani, S., Adhisivam, B., \& Bhat, B. V. (2016). Is maternal anxiety a barrier to exclusive breastfeeding?. The Journal of Maternal-Fetal \& Neonatal Medicine, 29(17), 2798-2801.

Association of Women's Health, Obstetric and Neonatal Nurses. (2014). AWHONN position statement: Breastfeeding. Journal of Obstetric, Gynecologic, and Neonatal Nursing, 44(1), 145-150.

Association of Women's Health, Obstetric, and Neonatal Nurses. (2015). Breastfeeding support: Preconception care through the first year (3rd ed.). Washington, DC: Author.

Caplinger, P., Cooney, A. T., Bledsoe, C., Hagan, P., Smith, A., Whitfield, P., ... Tipton, P. H. (2015). Breastfeeding outcomes following bariatric surgery. Clinical Lactation, 6(4), 144-152.

Centers for Disease Control and Prevention. (2016). Progress in increasing breastfeeding and reducing racial/ethnic differences: United States, 2000-2008 births. Retrieved from https://www.cdc.gov/breastfeeding/resources/breastfeeding-trends.htm

Centers for Disease Control and Prevention. (2018). Breastfeeding Report Card. Retrieved from http://www.cdc.gov.libproxy.usouthal.edu/breastfeeding/pdf/2014breastfeedingreportcard.pdf

Chantry, C. J., Eglash, A., \& Labbok, M. (2015). Position on breastfeeding-Revised 2015. Breastfeeding Medicine, 10(9), 407-411.

Chapman, D. J., \& Perez-Escamilla, R. (2013). Acculturative type is associated with breastfeeding duration among low-income Latinas. Maternal and Child Nutrition, 9, 188-198.

De Jager, E., Broadbent, J., Fuller-Tyszkiewicz, M., Nagle, C., McPhie, S., \& Skouteris, H. (2015). A longitudinal study of the effect of psychosocial factors on exclusive breastfeeding duration. Midwifery, 31(1), 103-111.

DiGirolamo, A. M., Grummer-Strawn, L. M., \& Fein, S. B. (2008). Effect of maternity-care practices on breastfeeding. Pediatrics, 122, S43-S49.

Dyson, L., McCormick, F. M., \& Renfrew, M. J. (2005). Interventions for promoting the initiation of breastfeeding. Cochrane Database of Systematic Reviews, (2), 1-42.

Feldman-Winter, L., Barone, L., Milcarek, B., Hunter, K., Meek, J., Morton, J., ... Lawrence, R. A. (2010). Residency curriculum improves breastfeeding care. Pediatrics, 126, 289-297.

Hawkins, S. S., Gillman, M. W., Shafer, E. F., \& Cohen, B. B. (2014). Acculturation and maternal health behaviors: Findings from the Massachusetts birth certificate. American Journal of Preventive Medicine, 47(2), 150-159.

Hedburg, I. C. (2013). Barriers to breastfeeding in the WIC population. Maternal-Child Nursing Journal, 38(4), 244-249.

Huang, Y., Ouyang, Y. Q., \& Redding, S. R. (2019). Previous breastfeeding experience and its influence on breastfeeding outcomes in subsequent births: A systematic review. Women and Birth, 32(4), 303-309.

Jansson, L. M., \& Velez, M. (2015). Lactation and the substance exposed mother-infant dyad. Journal of Perinatal and Neonatal Nursing, 29(4), 277-286.

Johnson, A. M., Kirk, R., \& Muzik, M. (2015). Overcoming workplace barriers: A focus group study exploring African American mothers' needs for workplace breastfeeding support. Journal of Human Lactation, 31(3), 425-433.

Kanhadilok, S., \& McGrath, J. M. (2015). An integrative review of factors influencing breastfeeding in adolescent 
mothers. Journal of Perinatal Education, 24(2), 119-127.

Khan, J., Vesel, L., Bahl, R., \& Martines, J. (2015). Timing of breastfeeding initiation and exclusivity of breastfeeding during the first month of life: Effects on neonatal mortality and morbidity-a systematic review and meta-analysis. Maternal \& Child Health Journal, 19(3), 468-479.

Kim, J. H., Fiese, B. H., \& Donovan, S. M. (2017). Breastfeeding is natural but not the cultural norm: A mixed-methods study of first-time breastfeeding, African-American mothers participating in WIC. Journal of Nutrition Education and Behavior, 49(7S2), S151-S161.

Labarere, J., Gelbert-Baudino, N., Ayral, A. S., Duc, C., Berchotteau, M., Bouchhon, N., ... Pons, J-C. (2005). Efficacy of breastfeeding support provided by trained clinicians during an early, routine, preventive visit: a prospective, randomized, open trial of 226 mother-infant pairs. Pediatrics, 115, 139-146.

Lepe, M., Bacardí, G. M., Castañeda-González, L. M., Pérez Morales, M. E., \& Jiménez, C. A. (2011). Effect of maternal obesity on lactation: Systematic review. Nutrición Hospitalaria, 26(6), 1266-1269.

Lind, J. N., Perrine, C. G., Li, R., Scanlon, K. S., \& Grummer-Strawn, L. M. (2014). Racial disparities in access to maternity care practices that support breastfeeding-United States. Retrieved from https://www.cdc.gov/mmwr/preview/mmwrhtml/mm6333a2.htm

Lumbiganon, P., Martis, R., Laopaiboon, M., Festin, M. R., Ho, J. J., \& Hakimi, M. (2012). Antenatal breastfeeding education for increasing breastfeeding duration. Cochrane Database of Systematic Reviews, (9), CD006425.

Majee, W., Jefferson, U. T., Goodman, L. R., \& Olsberg, J. E. (2016). Four years later: Rural mothers' and employers' perspectives on breastfeeding barriers following the passage of the Affordable Care Act. Journal of Health Care for the Poor and Underserved, 27(3), 1110-1125.

McKinney, C. O., Hahn-Holbrook, J., Chase-Lansdale, P. L., Ramey, S. L., Krohn, J., Reed-Vance, M., ... Shalowitz, M. U. (2016). Racial and ethnic differences in breastfeeding. Pediatrics, 138(2), 1-11.

Mueffelmann, R. E., Racine, E. F., Warren-Findlow, J., \& Coffman, M. J. (2015). Perceived infant feeding preferences of significant family members and mothers' intentions to exclusively breastfeed. Journal of Human Lactation, 31(3), 479-489.

Odom, E. C., Li, R., Scanlon, K. S., Perrine, C. G., \& Grummer-Strawn, L. (2013). Reasons for earlier than desired cessation of breastfeeding. Pediatrics, 131(3), e726-e732.

Perrine, C. G., Scanlon, K. S., Li, R., Odom, E., \& Grummer-Strawn, L. M. (2012). Baby-Friendly hospital practices and meeting exclusive breastfeeding intention. Pediatrics, 130, 54-60.

Reece-Stremtan, S., Marinelli, K. A., \& The Academy of Breastfeeding Medicine. (2015). ABM clinical protocol no 21: Guidelines for breastfeeding and substance use or substance use disorder. Breastfeeding Medicine, 10(3), 135-141.

Rempel, L. A., \& Rempel, J. K. (2011). The breastfeeding team: the role of involved fathers in the breastfeeding family. Journal of Human Lactation, 27, 115-121.

Roll, C. L., \& Cheater, F. (2016). Expectant parents' views of factors influencing infant feeding decisions in the antenatal period: A systematic review. International Journal of Nursing Studies, 60, 145-155.

Rosen-Carole, C., Hartman, S., \& Academy of Breastfeeding Medicine. (2015). ABM clinical protocol no. 19: Breastfeeding promotion in the prenatal setting. Breastfeeding Medicine, 10(10), 451-457.

Sankar, M. J., Sinha, B., Chowdhury, R., Bhandari, N., Taneja, S., Martines, J., \& Bahl, R. (2015). Optimal breastfeeding practices and infant and child mortality: A systematic review and meta-analysis. Acta Paediatrica, 104(467), 3-13.

Sipsma, H. L., Magriples, A., Gordon, D. D., Gabzdyl, E., \& Kershaw, T. (2013). Breastfeeding behavior among adolescents: Initiation, duration, and exclusivity. Journal of Adolescent Health, 53(3), 394-400.

Sparks, P. J. (2010). Rural-urban differences in breastfeeding initiation in the United States. Journal of Human Lactation, 26(2), 118-129.

Sparks, P. J. (2011). Racial/ethnic differences in breastfeeding duration among WIC-eligible families. Women's Health Issues, 21(5), 374-382.

Stamp, G. E., \& Casanova, H. T. (2006). A breastfeeding study in a rural population in South Australia. Rural Remote Health, 6(2), 495. 
Taveras, E. M., Capra, A. M., Braveman, P. A., Jensvold, N. G., Escobar, G. J., \& Lieu, T. A. (2013). Clinician support and psychosocial risk factors associated with breastfeeding discontinuation. Pediatrics, 112, 108-115.

Turcksin, R., Bel, S., Galjaard, S., \& Devlieger, R. (2014). Maternal obesity and breastfeeding intention, initiation, intensity and duration: A systematic review. Maternal and Child Nutrition, 10(2), 166-183.

U. S. Department of Health and Human Services. (2016). Healthy People 2020: Maternal, Infant, and Child Health. Washington, DC: Author. Retrieved from http://www.healthypeople.gov/2020/topics-objectives/topic/maternal-infant-and-child-health/objectives

Wambach, K., Domian, E. W., Page-Goertz, S., Wurtz, H., \& Hoffman, K. (2016). Exclusive breastfeeding experiences among Mexican American women. Journal of Human Lactation, 32(1), 103-111.

World Health Organization. (2016). Exclusive breastfeeding. E-library of evidence for nutrition actions. Geneva, Switzerland: Author. Retrieved from http://www.who.int/elena/titles/exclusive_breastfeeding/en/

\section{Copyrights}

Copyright for this article is retained by the author(s), with first publication rights granted to the journal.

This is an open-access article distributed under the terms and conditions of the Creative Commons Attribution license (http://creativecommons.org/licenses/by/4.0/). 